\title{
THE SINGLE-VALUED EXTENSION PROPERTY IS NOT PRESERVED UNDER SUMS AND PRODUCTS OF COMMUTING OPERATORS
}

\author{
A. BOURHIM \\ Département de Mathématiques et de Statistique, Université Laval, \\ Québec (Québec), Canada G1K 7 P4 \\ e-mail:bourhim@mat.ulaval.ca \\ and V. G. MILLER \\ Department of Mathematics and Statistics, Mississippi State University, \\ Mississippi State, MS 39762, USA \\ e-mail:vivien@math.msstate.edu
}

(Received 17 March, 2006; revised 21 August, 2006; accepted 3 August, 2006)

\begin{abstract}
We show that the sum and the product of two commuting operators with the single-valued extension property need not inherit this property.

2000 Mathematics Subject Classification. Primary 47B37. Secondary 47A10, 47A11.
\end{abstract}

1. Introduction. The problem of studying whether or not the local spectral properties such as the single-valued extension property, Dunford's condition $(C)$, Bishop's property $(\beta)$, the decomposition property $(\delta)$, or decomposability are preserved under sums and products of commuting operators has been considered by several authors, and remains an open problem; see for instance [3], [6], [9] and the references therein. Partial positive answers were obtained but only in certain special cases. In [9], T. L. Miller and M. M. Neumann showed that the sum and the product of two commuting operators with Dunford's condition $(C)$ have the single-valued extension property. They also proved that the product of two commuting operators has this property provided that one of them is non-invertible and has fat local spectra.

In this note, we show that the single-valued extension property is not preserved, in general, under the sums and products of commuting operators, and prove that the product of two commuting operators has this property provided that the intersection of their analytic cores is trivial. Our counter-examples are provided by tensor products of backward and forward unilateral weighted shifts, and our arguments and ideas are influenced by the ones given in [9].

We now gather together some basic facts about the single-valued extension property and local spectrum. Our reference is the excellent book of K. Laursen and M. M. Neumann [6].

Let $X$ be a complex Banach space, and let $\mathcal{L}(X)$ be the algebra of bounded linear operators on $X$. For an operator $T \in \mathcal{L}(X)$, we denote as usual its spectrum and its

The first author was supported by a CRM-ISM postdoctoral fellowship. The authors thank T. J. Ransford and T. L. Miller for useful comments and sincere encouragement. 
spectral radius by $\sigma(T)$ and $r(T):=\max \{|\lambda|: \lambda \in \sigma(T)\}$. By Gelfand's formula for the spectral radius $r(T)=\lim _{n \rightarrow+\infty}\left\|T^{n}\right\|^{\frac{1}{n}}$ for all $T \in \mathcal{L}(X)$.

An operator $T \in \mathcal{L}(X)$ is said to have the single-valued extension property provided that for every open subset $U$ of $\mathbb{C}$ the only analytic solution $\phi: U \rightarrow X$ of the equation

$$
(T-\lambda) \phi(\lambda)=0,(\lambda \in U)
$$

is the zero function. The local resolvent set, $\rho_{T}(x)$, of an operator $T \in \mathcal{L}(X)$ at a point $x \in X$ is the union of all open subsets $U$ of $\mathbb{C}$ for which there is an analytic function $\phi: U \rightarrow X$ that satisfies $(T-\lambda) \phi(\lambda)=x \quad(\lambda \in U)$. The local spectrum of $T$ at $x$ is defined by $\sigma_{T}(x):=\mathbb{C} \backslash \rho_{T}(x)$, and is obviously a closed subset of $\sigma(T)$.

We conclude this introduction by providing some basic definitions about tensor products quoted from [6, Chapter 2]. Let $\mathcal{H}_{1}$ and $\mathcal{H}_{2}$ be complex Hilbert spaces with the scalar products $\langle,\rangle_{1}$ and $\langle,\rangle_{2}$, respectively. Let $\mathcal{H}=\mathcal{H}_{1} \otimes \mathcal{H}_{2}$ be the Hilbert tensor product of $\mathcal{H}_{1}$ and $\mathcal{H}_{2}$ whose scalar product is determined by the formula

$$
\left\langle h_{1} \otimes h_{2}, k_{1} \otimes k_{2}\right\rangle:=\left\langle h_{1}, k_{1}\right\rangle_{1}\left\langle h_{2}, k_{2}\right\rangle_{2},\left(h_{1}, k_{1} \in \mathcal{H}_{1}, h_{2}, k_{2} \in \mathcal{H}_{2}\right) .
$$

The tensor product of two operators $A \in \mathcal{L}\left(\mathcal{H}_{1}\right)$ and $B \in \mathcal{L}\left(\mathcal{H}_{2}\right)$ is determined by the formula $(A \otimes B)\left(h_{1} \otimes h_{2}\right):=A h_{1} \otimes B h_{2},\left(h_{1} \in \mathcal{H}_{1}, h_{2} \in \mathcal{H}_{2}\right)$, and its spectral radius is given by

$$
\begin{aligned}
r(A \otimes B) & =\lim _{n \rightarrow+\infty}\left\|(A \otimes B)^{n}\right\|^{\frac{1}{n}}=\lim _{n \rightarrow+\infty}\left\|A^{n} \otimes B^{n}\right\|^{\frac{1}{n}} \\
& =\lim _{n \rightarrow+\infty}\left(\left\|A^{n}\right\|\left\|B^{n}\right\|\right)^{\frac{1}{n}}=r(A) r(B) .
\end{aligned}
$$

Thus, if either $A$ or $B$ is quasinilpotent, then so is $A \otimes B$.

2. Counter-examples. Let $l^{2}\left(\mathbb{Z}^{+}\right)$be the usual Hilbert space of all square summable complex sequences. For a positive bounded sequence $\alpha:=\left(\alpha_{n}\right)_{n \geq 0}$, let $S_{\alpha}$ be the corresponding weighted unilateral shift on $l^{2}\left(\mathbb{Z}^{+}\right)$defined by

$$
S_{\alpha} x:=\left(0, \alpha_{0} x_{0}, \alpha_{1} x_{1}, \alpha_{2} x_{2}, \ldots\right), \quad x=\left(x_{n}\right)_{n \geq 0} \in l^{2}\left(\mathbb{Z}^{+}\right) .
$$

Its adjoint is given by

$$
S_{\alpha}^{*} x:=\left(\alpha_{0} x_{1}, \alpha_{1} x_{2}, \alpha_{2} x_{3}, \ldots\right), \quad x=\left(x_{n}\right)_{n \geq 0} \in l^{2}\left(\mathbb{Z}^{+}\right) .
$$

It has the single-valued extension property if and only if

$$
R\left(S_{\alpha}\right):=\liminf _{n \rightarrow+\infty}\left(\alpha_{0} \alpha_{1} \ldots \alpha_{n-1}\right)^{\frac{1}{n}}=0
$$

see for instance [8, Proposition 2.5].

We need the following elementary lemma.

Lemma 2.1. Assume that $\alpha:=\left(\alpha_{n}\right)_{n \geq 0}$ and $\beta:=\left(\beta_{n}\right)_{n \geq 0}$ are two positive bounded sequences. The following hold.

(a) The operator $S_{\beta}^{*} \otimes 1$ has the single-valued extension property if and only if $R\left(S_{\beta}\right)=0$.

(b) $\left\{\lambda \in \mathbb{C}:|\lambda| \leq R\left(S_{\alpha}\right)\right\} \subset \sigma_{1 \otimes S_{\alpha}}(z)$ for all nonzero elements $z$ of $l^{2}\left(\mathbb{Z}^{+}\right) \otimes l^{2}\left(\mathbb{Z}^{+}\right)$. 
Proof. Observe that $S_{\beta}^{*} \otimes 1$ and $1 \otimes S_{\alpha}$ are unitarily equivalent to $\sum_{n \geq 0} \oplus S_{\beta}^{*}$ and $\sum_{n \geq 0} \oplus S_{\alpha}$, respectively, and use for instance [4, Proposition 3.1] or [8, Proposition 2.6].

THEOREM 2.2. For two positive bounded sequences $\alpha:=\left(\alpha_{n}\right)_{n \geq 0}$ and $\beta:=\left(\beta_{n}\right)_{n \geq 0}$, the operator $S_{\beta}^{*} \otimes\left(S_{\alpha}-a\right)$ has the single-valued extension property for all $a \in \mathbb{C},|a| \leq$ $R\left(S_{\alpha}\right)$.

Proof. If $S_{\beta}^{*}$ is quasinilpotent then so is $S_{\beta}^{*} \otimes\left(S_{\alpha}-a\right)$. Thus, we may assume that $S_{\beta}^{*}$ is not quasinilpotent. Dividing $S_{\beta}^{*}$ by $r\left(S_{\beta}^{*}\right)$, if necessary, we can also suppose that $r\left(S_{\beta}^{*}\right)=1$. Now, let $\phi: U \rightarrow l^{2}\left(\mathbb{Z}^{+}\right) \otimes l^{2}\left(\mathbb{Z}^{+}\right)$be an analytic function such that

$$
\left(S_{\beta}^{*} \otimes\left(S_{\alpha}-a\right)-\lambda\right) \phi(\lambda)=0,(\lambda \in U),
$$

and let us prove that $\phi$ is identically zero in $U$. Pick a nonzero $\lambda_{0} \in U$, and let $D:=$ $\left\{\lambda \in \mathbb{C}:|\lambda|<\left|\lambda_{0}\right|\right\}$. Let

$$
\Phi(\lambda):=\left(\left(\lambda_{0}-\lambda S_{\beta}^{*}\right)^{-1} S_{\beta}^{*} \otimes 1\right) \phi\left(\lambda_{0}\right),(\lambda \in D) .
$$

For every $\lambda \in D$, the operators $\left(S_{\beta}^{*} \otimes\left(S_{\alpha}-a\right)-\lambda_{0}\right),\left(\left(\lambda_{0}-\lambda S_{\beta}^{*}\right)^{-1} \otimes 1\right)$ and $S_{\beta}^{*} \otimes 1$ commute with each other, and

$$
\begin{aligned}
\phi\left(\lambda_{0}\right) & =0+\left(\lambda_{0}-\lambda S_{\beta}^{*} \otimes 1\right)\left(\left(\lambda_{0}-\lambda S_{\beta}^{*}\right)^{-1} \otimes 1\right) \phi\left(\lambda_{0}\right) \\
& =\left[\left[\left(S_{\beta}^{*} \otimes\left(S_{\alpha}-a\right)-\lambda_{0}\right)+\left(\lambda_{0}-\lambda S_{\beta}^{*} \otimes 1\right)\right]\left(\left(\lambda_{0}-\lambda S_{\beta}^{*}\right)^{-1} \otimes 1\right)\right] \phi\left(\lambda_{0}\right) \\
& =\left[\left[\left(S_{\beta}^{*} \otimes\left(S_{\alpha}-a\right)-\lambda S_{\beta}^{*} \otimes 1\right)\right]\left(\left(\lambda_{0}-\lambda S_{\beta}^{*}\right)^{-1} \otimes 1\right)\right] \phi\left(\lambda_{0}\right) \\
& =\left(1 \otimes\left(S_{\alpha}-a\right)-\lambda\right)\left(S_{\beta}^{*} \otimes 1\right)\left(\left(\lambda_{0}-\lambda S_{\beta}^{*}\right)^{-1} \otimes 1\right) \phi\left(\lambda_{0}\right) .
\end{aligned}
$$

This shows that

$$
\left(1 \otimes\left(S_{\alpha}-a\right)-\lambda\right) \Phi(\lambda)=\phi\left(\lambda_{0}\right)
$$

for all $\lambda \in D$, and $a \in\{a\}+D \subset \rho_{1 \otimes S_{\alpha}}\left(\phi\left(\lambda_{0}\right)\right)$. As $|a| \leq R\left(S_{\alpha}\right)$, we see that $\phi\left(\lambda_{0}\right)=0$ by Lemma 2.1-(b). Since $\lambda_{0}$ is an arbitrary nonzero element of $U$, we see that $\phi \equiv 0$, and $S_{\beta}^{*} \otimes\left(S_{\alpha}-a\right)$ has the single-valued extension property; as desired.

Assume that $\alpha:=\left(\alpha_{n}\right)_{n \geq 0}$ and $\beta:=\left(\beta_{n}\right)_{n \geq 0}$ are two positive bounded sequences such that $R\left(S_{\alpha}\right) \geq 1$ and $R\left(S_{\beta}\right)>0$. Let

$$
R:=S_{\beta}^{*} \otimes\left(S_{\alpha}+1\right) \text { and } T:=S_{\beta}^{*} \otimes\left(1-S_{\alpha}\right) .
$$

Obviously, $R$ and $T$ commute, and have the single-valued extension property by Theorem 2.2. But

$$
R+T=2 S_{\beta}^{*} \otimes 1
$$

is without this property (see Lemma 2.1-(a)).

Note that $R_{1}:=\exp (R)$ and $T_{1}:=\exp (T)$ commute and have the single-valued extension property as well since this property is invariant under the analytic functional calculus; see [6, Theorem 3.3.9]. But $R_{1} T_{1}=\exp (R+T)$ does not have this property since $R+T$ does not. 
3. The role of the analytic core. For an operator $T \in \mathcal{L}(X)$, the analytic core is defined by

$$
\begin{array}{r}
K(T):=\left\{x \in X: \text { there exist } a>0 \text { and a sequence }\left(x_{n}\right)_{n \geq 1} \in X\right. \text { such that } \\
\left.\qquad x_{1}=x, T x_{n+1}=x_{n} \text { and }\left\|x_{n}\right\| \leq a^{n}\|x\| \text { for all } n \geq 1\right\} .
\end{array}
$$

It easily follows from the definition that $K(T)$ is a linear subspace of $X$ and that $T(K(T))=K(T)$; see [7].

The following lemma is needed.

LeMma 3.1. For every operator $T \in \mathcal{L}(X)$, we have

$$
K(T)=\left\{x \in X: 0 \in \rho_{T}(x)\right\} .
$$

Proof. See [7, Proposition 1.3-(a)].

The following result generalizes [9, Theorem 3].

THEOREM 3.2. Assume that $T, S \in \mathcal{L}(X)$ are two commuting operators. If $K(T) \cap$ $K(S)=\{0\}$, then $T S$ has the single-valued extension property.

Proof. Note that, since $r(T S) \leq r(T) r(S)$, the product $T S$ is quasinilpotent provided that either $T$ or $S$ is quasinilpotent. Thus, we may and shall assume that $r(T)=r(S)=1$. Let $\phi: U \rightarrow X$ be an analytic function such that

$$
(T S-\lambda) \phi(\lambda)=0,(\lambda \in U),
$$

and let us prove that $\phi$ is identically zero in $U$. Pick a nonzero $\lambda_{0} \in U$, and set $D:=\left\{\lambda \in \mathbb{C}:|\lambda|<\left|\lambda_{0}\right|\right\}$. Let us consider the following $X$-valued analytic function in $D$,

$$
\Phi(\lambda):=\left(\lambda_{0}-\lambda T\right)^{-1} T \phi\left(\lambda_{0}\right),(\lambda \in D) .
$$

For every $\lambda \in D$, we have

$$
\begin{aligned}
(S-\lambda) \Phi(\lambda) & =\left(\lambda_{0}-\lambda T\right)^{-1}(S T-\lambda T) \phi\left(\lambda_{0}\right) \\
& =\left(\lambda_{0}-\lambda T\right)^{-1}\left[\left(S T-\lambda_{0}\right)+\left(\lambda_{0}-\lambda T\right)\right] \phi\left(\lambda_{0}\right) \\
& =\phi\left(\lambda_{0}\right) .
\end{aligned}
$$

This shows that $D \subset \rho_{S}\left(\phi\left(\lambda_{0}\right)\right)$, and $\phi\left(\lambda_{0}\right) \in K(S)$ by Lemma 3.1.

A similar argument shows that $\phi\left(\lambda_{0}\right) \in K(T)$ as well. As $K(T) \cap K(S)=\{0\}$, we see that $\phi\left(\lambda_{0}\right)=0$ and $\phi$ vanishes, in fact, on the whole of $U$. This proves that $T S$ has the single-valued extension property.

The following corollaries are immediate consequences of Theorem 3.2.

COROllary 3.3. Assume that $S \in \mathcal{L}(X)$ satisfies the abstract shift condition; i.e., $\bigcap_{n \geq 0} S^{n} X=\{0\}$. Then, for every operator $T \in \mathcal{L}(X)$ commuting with $S$, the product $T S$ has the single-valued extension property.

Proof. As $S(K(S))=K(S)$, we see that $K(S) \subset \bigcap_{n \geq 0} S^{n} X=\{0\}$.

An operator $T \in \mathcal{L}(X)$ is said to have fat local spectra provided that $\sigma_{T}(x)=\sigma(T)$ for all nonzero $x \in X$. 
COROllary 3.4 ([9, Theorem 3]). Let $S \in \mathcal{L}(X)$ be a non-invertible operator with fat local spectra. Then, for every operator $T \in \mathcal{L}(X)$ commuting with $S$, the product $T S$ has the single-valued extension property.

Proof. We have $0 \in \sigma(S)=\sigma_{S}(x)$ for all nonzero $x \in X$, and $K(S)=\{0\}$.

We finally note that under the conditions of Theorem 3.2, the sum $T+S$ need not have the single-valued extension property. Indeed, assume that $\alpha:=\left(\alpha_{n}\right)_{n \geq 0}$ and $\beta:=$ $\left(\beta_{n}\right)_{n \geq 0}$ are two positive bounded sequences such that $\lim _{n \rightarrow+\infty} \alpha_{n}=0$ and $R\left(S_{\beta}\right)>0$, and let $T:=1 \otimes S_{\alpha}$ and let $S:=S_{\beta}^{*} \otimes 1$. The following facts hold trivially:

(a) $T S=S T$.

(b) $T$ is quasinilpotent and $K(T)=\{0\}$ since $\lim _{n \rightarrow+\infty} \alpha_{n}=0$.

(c) $S$ is without the single-valued extension property since $R\left(S_{\beta}\right)>0$.

By (a) and (b), we see that $T+S$ and $S$ are quasinilpotent equivalent; see [6, Page 253]. As quasinilpotent equivalence preserves the single-valued extension property, it follows that $T+S$ is without this property; see [6, Proposition 3.4.11].

4. Remarks and open problems. The question of which properties are preserved under tensor products has been studied by many authors and turns out to be very complicated. It is known that the tensor product $A \otimes B$ of two operators $A \in \mathcal{L}\left(\mathcal{H}_{1}\right)$ and $B \in \mathcal{L}\left(\mathcal{H}_{2}\right)$ is normal (resp. quasinormal, subnormal, hyponormal, $p$-hyponormal, loghyponormal) if and only if so are $A$ and $B$; see [5], [10], [11], [12]. However, it may happen that $A \otimes B$ has a property but $A$ and $B$ do not mainly because $A \otimes B=(c A) \otimes\left(c^{-1} B\right)$ for all nonzero $c \in \mathbb{C}$. It may also happen that both $A$ and $B$ have a property while $A \otimes B$ does not. For example this happens in the case when both $A$ and $B$ are paranormal operators; see [10, Page 629].

A similar natural problem for local spectral properties suggests itself.

Problem 1. Asumme that $A \in \mathcal{L}\left(\mathcal{H}_{1}\right)$ and $B \in \mathcal{L}\left(\mathcal{H}_{2}\right)$ are two operators satisfying a local spectral property such as the single-valued extension property, Dunford's condition $(C)$, Bishop's property $(\beta)$, the decomposition property $(\delta)$, or decomposability. Does $A \otimes B$ satisfy the same property?

It may happen that $A \otimes B$ has a local spectral property though $A$ or $B$ do not mainly because $A \otimes B$ is quasinilpotent provided that one of them is quasinilpotent. However, it was shown in [2, Corollary 2.5] that if $A$ and $B$ are decomposable, then so is $A \otimes B$. This result together with [6, Theorem 2.4.4 and Theorem 2.5.5] shows, in fact, that even Bishop's property $(\beta)$ and the decomposition property $(\delta)$ are preserved under Hilbert tensor product of two operators. But the question of whether $A \otimes B$ has the single-valued extension property or Dunford's condition $(C)$ whenever $A$ and $B$ have the same property is far away from being clear. However, if $A \otimes B$ has the single-valued extension property then either $A$ or $B$ has this property. Indeed, assume by the way of contradiction that there is an analytic $\mathcal{H}_{i}$-valued function $\phi_{i}$ in an open subset $U_{i}$ of $\mathbb{C},(i=1,2)$, such that

$$
(A-\lambda) \phi_{1}(\lambda)=0,\left(\lambda \in U_{1}\right), \text { and }(B-\lambda) \phi_{2}(\lambda)=0,\left(\lambda \in U_{2}\right) .
$$

Pick a nonzero $\lambda_{1} \in U_{1}$ for which $x:=\phi_{1}\left(\lambda_{1}\right) \neq 0$, and set

$$
\phi(z):=x \otimes \phi_{2}\left(z / \lambda_{1}\right),\left(z \in U:=\lambda_{1} U_{2}\right) .
$$


It is an analytic $\mathcal{H}_{1} \otimes \mathcal{H}_{2}$-valued function in the open subset $U$. For every $z \in U$, we have

$$
\begin{aligned}
(A \otimes B-z) \phi(z) & =(A \otimes B-z) x \otimes \phi_{2}\left(z / \lambda_{1}\right) \\
& =\left[\left(A-\lambda_{1}\right) x\right] \otimes B \phi_{2}\left(z / \lambda_{1}\right)+\lambda_{1} x \otimes\left[\left(B-z / \lambda_{1}\right) \phi_{2}\left(z / \lambda_{1}\right)\right] \\
& =0+0=0 .
\end{aligned}
$$

This contradicts the fact that $A \otimes B$ has the single-valued extension property.

To close this paper with the final remark, we need to fix some notation. For an operator $T \in \mathcal{L}(X)$, we denote by $\Re(T)$ the set of all complex numbers where $T$ fails to have the single-valued extension property; see [1]. It is an open subset of $\mathbb{C}$ contained in the point spectrum $\sigma_{p}(T)$ of $T$, and is empty precisely when $T$ has the single-valued extension property.

REMARK 4.1. For two subsets $\Lambda_{1}$ and $\Lambda_{2}$ of $\mathbb{C}$, we denote $\Lambda_{1} \cdot \Lambda_{2}:=\left\{\lambda_{1} \lambda_{2}: \lambda_{i} \in\right.$ $\left.\Lambda_{i},(i=1,2)\right\}$. The above arguments show, in fact, that

$$
\left\{\sigma_{p}(A) \backslash\{0\}\right\} . \Re(B) \cup \Re(A) .\left\{\sigma_{p}(B) \backslash\{0\}\right\} \subset \Re(A \otimes B)
$$

for all $A \in \mathcal{L}\left(\mathcal{H}_{1}\right)$ and $B \in \mathcal{L}\left(\mathcal{H}_{2}\right)$.

\section{REFERENCES}

1. P. Aiena and O. Monsalve, Operators which do not have the single valued extension property, J. Math. Anal. Appl. 250 (2000), 435-448.

2. E. Albrecht, J. Eschmeier and M. M. Neumann, Some topics in the theory of decomposable operators, Oper. Theory Adv. Appl. 17 (1986), 15-34.

3. B. Aupetit and D. Drissi, Local spectrum and subharmonicity, Proc. Edinburgh Math. Soc. (2) 39 (1996), 571-579.

4. A. Bourhim, On the local spectral properties of weighted shift operators, Studia Math. 163 (2004), no. 1, 41-69.

5. B. P. Duggal, Tensor products of opertors-strong stability and p-hyponormality, Glasgow Math. J. 42 (2000), 371-381.

6. K. B. Laursen and M. M. Neumann, An introduction to local spectral theory, London Mathematical Society Monograph New Series No. 20 (Oxford University Press).

7. M. Mbekhta, Sur la théorie spectrale locale et limite des nilpotents, Proc. Amer. Math. Soc. 110 (1990), 621-631.

8. T. L. Miller, V. G. Miller and M. M. Neumann, Local spectral properties of weighted shifts, J. Operator Theory 51 (2004), no. 1, 71-88.

9. T. L. Miller and M. M. Neumann, The single-valued extension property for sums and products of commuting operators, Czechoslovak Math. J. 52(127) (2002), no. 3, 635-642.

10. T. Saito, Hyponormal operators and related topics, Lecture Notes in Mathematics, vol. 247 (Springer-Verlag, 1971).

11. J. Stochel, Seminormality of operators from their tensor products, Proc. Amer. Math. Soc. 124 (1996), 435-440.

12. K. Tanahashi and M. Chō, Tensor products of log-hyponormal and of class $A(s, t)$ operators, Glasgow. Math. J. 46 (2004), 91-95. 